Physics, Chemistry, and Dynamics of Interplanetary Dust

ASP Conference Series, Vol. 104, 1996

Bo A. S. Gustafson and Martha S. Hanner (eds.)

\title{
Sources of 10 micron Interplanetary Dust: The Contribution from the Kuiper Belt
}

\author{
G. J. Flynn \\ Dept. of Physics, SUNY-Plattsburgh, Plattsburgh, NY 12901
}

\begin{abstract}
Two major sources are observed to contribute to the Zodiacal Cloud: main-belt asteroids and active comets. However, the discovery of $100 \mathrm{~km}$ size objects in trans-Neptunian orbits (Jewitt \& Luu 1993) coupled with Pioneer 10 measurements showing an essentially constant flux of $10 \mu \mathrm{m}$ dust from 4 to $18 \mathrm{AU}$ with indications that dust may be in near-circular orbits (Humes 1980) suggests that collisions in the Kuiper Belt may contribute significantly to the Zodiacal Cloud and to the interplanetary dust collected from the Earth's stratosphere. Kuiper Belt dust collected at Earth could be identified by high densities of solar flare tracks, unusually thick amorphous rims, and high concentrations of spallogenic isocopes.
\end{abstract}

\section{Introduction}

Dust particles are removed from interplanetary space by Poynting-Robertson drag (P-R drag) and by catastrophic collisions on time scales that are short compared to the age of the solar system. Thus, a recent source is required to produce the current interplanetary dust complex. In principle every solar system object can contribute to the interplanetary dust cloud by processes such as outgassing, erosion, catastrophic collisions, tidal disruption, and volcanism. However, active comets and main-belt asteroids are assumed to be the major contributors to the Zodiacal Cloud because of the infrared observations of dust trails or dust bands associated with these sources.

\section{Constraints on the Sources}

Dermott et al. (1994), from detailed modeling of the infrared dust bands, find that the main-belt asteroids contribute about $30 \%$ of the dust to the Zodiacal Cloud. The remainder of the cloud requires a source with a higher mean inclination to the ecliptic. Dermott et al. (1994) suggest active comets are the likely source for the remaining $70 \%$ of the Zodiacal dust. However, Flynn (1992) pointed out that a cometary source of this magnitude should result in catastrophic collision lifetimes for large $(>200 \mu \mathrm{m})$ micrometeorites which are too short to allow their survival during transport by P-R drag from the mainbelt to the Earth. The collection of large micrometeorites having cosmic ray exposure durations consistent with a main-belt asteroidal origin (Nishiizumi et 
al. 1991) suggests that the contribution of active comets to the Zodiacal Cloud is significantly smaller than $70 \%$ of the total (Flynn 1992).

Taken together these results suggest a third contributor to the Zodiacal Cloud which provides dust in nearly circular orbits but with a larger range of inclinations than the dust provided by main-belt asteroids, and which is undetected by satellite infrared measurements, presumably because of its large heliocentric distance. Debris produced by collisions in the Kuiper Belt would meet these criteria (Flynn 1994).

\section{Evidence From the Pioneer Spacecraft}

The Pioneer 10 and Pioneer 11 spacecraft detected dust particles out to about $18 \mathrm{AU}$ (Humes 1980), where the dust detectors ceased to function. This direct measurement of interplanetary dust with masses $>8 \times 10^{-10} \mathrm{~g}(\sim 9 \mu$ m diameter $)$ by Pioneer 10 and $>6 \times 10^{-9} \mathrm{~g}(\sim 18 \mu \mathrm{m}$ diameter $)$ by Pioneer 11 provides observational evidence of the orbital properties of the dust and constraints on its major sources. The Pioneer measurements have been interpreted to indicate that:

1) a large fraction of the dust at $1 \mathrm{AU}$ is in near-circular orbits (Humes 1974), 2) a large fraction of the dust near Jupiter (5.2 AU) and Saturn (9.5 AU) may be in near-circular heliocentric orbits (Humes 1980), and,

3 ) that the rate of dust impacts does not diminish significantly after passage through the main-belt, remaining roughly constant out to $18 \mathrm{AU}$ (Humes 1980).

The first two observations suggest that active comets are not the dominant source of interplanetary dust, since the eccentricities of the orbits of cometary dust particles are still significant at 1 AU (Flynn 1989; Jackson \& Zook 1992). The third observation suggests that main-belt asteroids are not the dominant source of interplanetary dust, since P-R drag would cause the orbits of $10 \mu \mathrm{m}$ dust produced in the main-belt to evolve rapidly inward, resulting in a sharp drop in the dust detection rate outside of the main- belt. The Pioneer measurements are consistent with a significant dust source at a heliocentric distance $\gg 4 \mathrm{AU}$, producing interplanetary dust in near-circular orbits.

\section{A Kuiper Belt Source of the Pioneer 10/11 Dust}

The discovery by Jewitt \& Luu (1993) of large objects $(>100 \mathrm{~km})$ in transNeptunian, heliocentric orbits possibly provided the first detection of the Kuiper Belt, the proposed source for short-period comets. Jewitt \& Luu (1995) identified 24 trans-Neptunian objects, and estimated the number of undetected objects $>100 \mathrm{~km}$ as $\sim 10^{4}$ A Hubble Space Telescope search for smaller objects identified 29 Kuiper Belt objects of $\sim 10 \mathrm{~km}$ diameter, with an estimated total population of $\sim 2 \times 10^{8}$ such objects within $40 \mathrm{AU}$ and having inclinations up to $12^{\circ}$ (Cochran et al. 1995). Collisions between these objects should result in significant dust production in the Kuiper Belt.

Once the size-frequency distribution and the spatial density of the objects in the Kuiper Belt are established, the dust production rate from collisions within the belt can be modeled. At present, we can use the Pioneer measurements to estimate the contribution at Earth from the dust detected at $18 \mathrm{AU}$. 


\section{Modeling the Pioneer 10 and 11 Flux at Earth}

Since the Pioneer spacecraft detected a relatively constant flux of dust from 4 to $18 \mathrm{AU}$ (except near Jupiter and Saturn), the simplest model of the terrestrial contribution from the dust detected at $18 \mathrm{AU}$ assumes that there is no significant sink for this dust inside of $4 \mathrm{AU}$, and the flux remains constant to $1 \mathrm{AU}$. An obvious mechanism for a dust sink would be vaporization, if the meteoroids were made predominantly of ices.

In the absence of a sink, the contribution from the dust detected at $18 \mathrm{AU}$ to the flux of dust particles having masses $8 \times 10^{-10} \mathrm{~g}$ at Earth would be $2 \times 10^{-6}$ particles $/ \mathrm{m}^{2} \cdot \mathrm{s}$. Since the total flux of micrometeoroids $>10^{-9} \mathrm{~g}$ measured in low-Earth orbit, on the Long Duration Exposure Facility, is $1 \times 10^{-5}$ particles $/ \mathrm{m}^{2} \cdot \mathrm{s}$ (Love \& Brownlee 1993), the particles detected by Pioneer 10 at 18 AU could contribute $\sim 20 \%$ of the total $\sim 10 \mu \mathrm{m}$ diameter interplanetary dust at Earth.

This simple model overlooks four important considerations;

1) the expected increase in spatial density with decreasing heliocentric distance for particles evolving into the Sun under the influence of only P-R drag and solar gravity,

2) gravitational focusing which increases the effective capture cross-section,

3) particle loss by gravitational interactions with Jupiter and Saturn, and,

4) particle loss (or production) by catastrophic collisions.

The first two factors increase the dust flux at Earth, while the last two decrease the flux over that measured at 18 AU. Liou et al. (1995) have modeled the orbital evolution of small dust particles from the Kuiper Belt. They found that gravitational scattering by the gas giant planets ejects about $80 \%$ of these particles from the solar system, while the remaining $20 \%$ reach $1 \mathrm{AU}$ with relatively low orbital eccentricities.

Assuming: 1) an $80 \%$ loss of dust encountering the gas giants, 2) an Earth encounter velocity of $5 \mathrm{~km} / \mathrm{s}$, and 3) orbital evolution under the influence of P-R drag, the particles detected by Pioneer 10 at 18 AU should contribute $2 \times 10^{-5}$ particles $/ \mathrm{m}^{2}$.s at Earth, which exceeds the measured terrestrial flux of particles $>10^{-9} \mathrm{~g}$ by a factor of 2 . This clearly overestimates the actual contribution.

Liou et al. (1996) point out that dust grains $>4 \mu \mathrm{m}$ in diameter have a high probability of loss by catastrophic collisions with interstellar dust while evolving from $40 \mathrm{AU}$ into the inner solar system. The relatively constant dust flux measured by Pioneer 10 from 18 AU to 4 AU suggests significant particle loss (by collisions or by planetary scattering), since orbital modeling using only $\mathrm{P}-\mathrm{R}$ drag indicates that the flux should increase by about an order-of-magnitude from $18 \mathrm{AU}$ to $4 \mathrm{AU}$. However the Pioneer data also demonstrate that, unless there are additional dust sources between $18 \mathrm{AU}$ and $4 \mathrm{AU}$, a significant fraction $(\sim 10 \%)$ of the $\sim 10 \mu \mathrm{m}$ dust detected at $18 \mathrm{AU}$ survives to reach $4 \mathrm{AU}$.

\section{Identification of IDPs From the Kuiper Belt}

Ten micron diameter IDPs of density $2 \mathrm{~g} / \mathrm{cm}^{3}$ which originate in the Kuiper Belt ( $40 \mathrm{AU})$ and spiral inward under P-R drag without experiencing either fragmentation or trapping in a gravitional resonance reach $1 \mathrm{AU}$ in $\sim 1 \times 10^{7}$ 


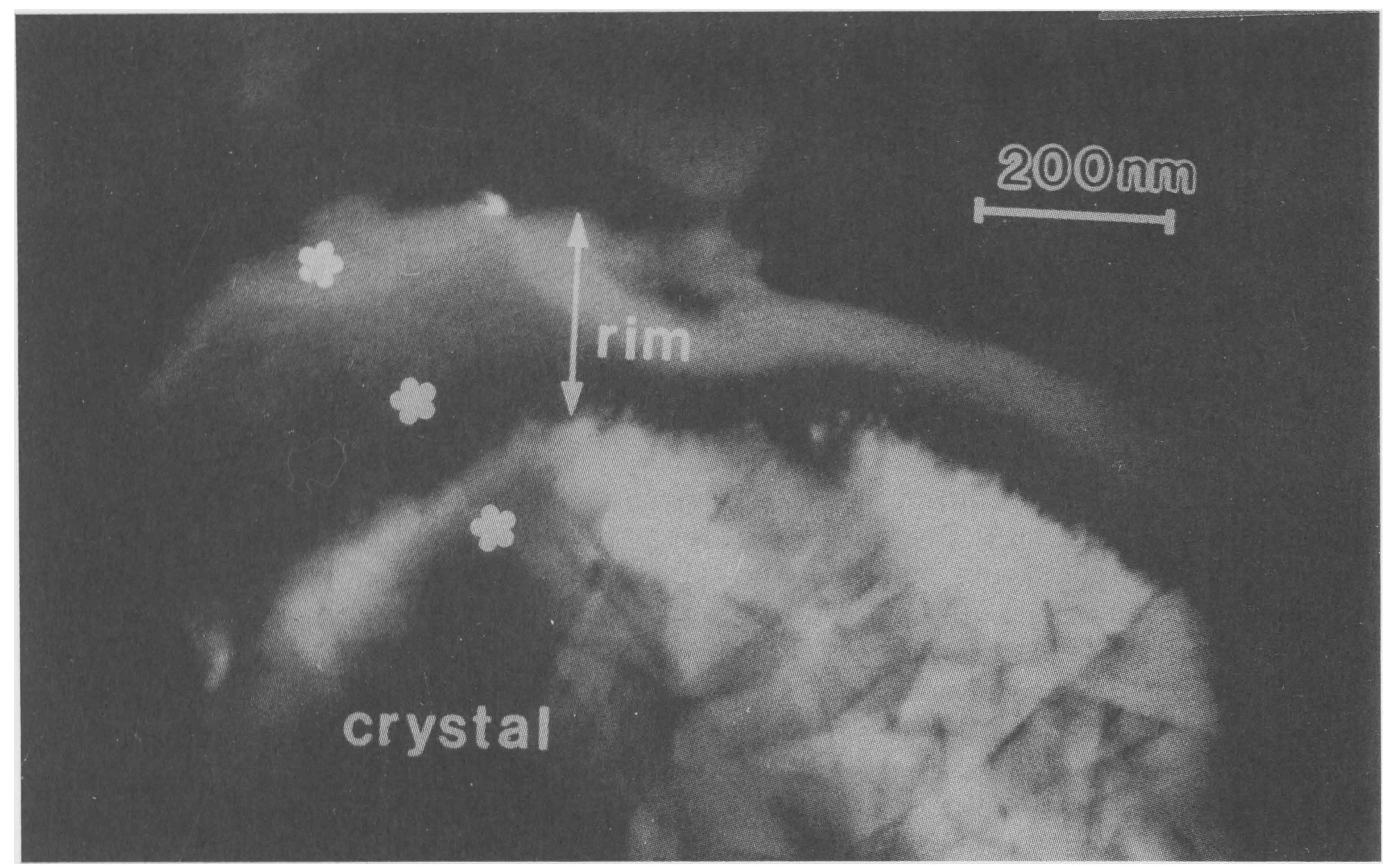

Figure 1. TEM image of one edge of W7027 C4, a 12 micron interplanetary dust particle (IDP) collected from the Earth's stratosphere, which has an order-of-magnitude higher solar flare track density (dark, linear features in the crystal) and a much thicker amorphous rim $(\sim 200 \mathrm{~nm})$ than do typical IDPs. (Photo by J. P. Bradley.)

years, compared to $\sim 6 \times 10^{4}$ years for similar particles spiraling from the mainbelt to $1 \mathrm{AU}$. The duration of space exposure for cometary IDPs depends on the initial orbit, but the space exposure ages of typical $10 \mu \mathrm{m}$ cometary IDPs range from $3 \times 10^{4}$ years (d'Arrest) to $11 \times 10^{4}$ years (Swift-Tuttle) (Flynn 1989). Thus, IDPs from the Kuiper Belt experience significantly greater effects of space exposure than do main-belt asteroidal or cometary IDPs. The effects of space exposure are the production of nuclear damage tracks, amorphous rims on exterior surfaces, and cosmogenic nuclides.

Nuclear damage tracks, imaged in a Transmission Electron Microscope (TEM), are seen in the crystaline phases of IDPs recovered from the Earth's stratosphere. The track densities are generally consistent with the space exposure received in traveling from the main-belt to the Earth under P-R drag (Sandford \& Bradley 1990). Assuming: 1) a $1 / r^{2}$ falloff of solar flare Fe-ion flux with heliocentric distance $r, 2$ ) orbital evolution dominated by P-R drag, and 3) no particle fragmentation enroute, the track density in an IDP originating in the Kuiper Belt would exceed that in main-belt asteroidal IDPs of the same size and density by a factor of $\sim 12$.

Solar wind ions, which have energies of $\sim 1 \mathrm{KeV} /$ nucleon, are implanted to depths of tens of nanometers in crystals exposed on the lunar surface. This produces amorphous, radiation damaged rims typically $\sim 30 \mathrm{~nm}$ thick (Bibring et 
al. 1972). Similar amorphous rims, with thicknesses up to $50 \mathrm{~nm}$, are seen on the exposed surfaces of olivine and pyroxene grains on the exterior of IDPs (Bradley \& Brownlee 1986). Higher energy solar particles, $>10 \mathrm{KeV} /$ nucleon, are less abundant, but given long exposure times $(>100,000$ years) these solar energetic particles should produce amorphous rims 150 to $250 \mathrm{~nm}$ thick (Christopherson \& McKay 1995). Thus, IDPs from the Kuiper Belt should have amorphous rims $250 \mathrm{~nm}$ thick, about a factor of 5 thicker than the rims on IDPs from main-belt asteroids or from typical active comets.

Spallogenic nuclei are produced by galactic and solar cosmic rays. Kuiper Belt IDPs would receive $\sim 300$ times the integrated dose from galactic cosmic rays and $\sim 12$ times the integrated dose from solar cosmic rays as do IDPs from the main-belt.

Bradley has identified one IDP, out of about 50 examined in detail, with a solar flare track density about an order-of-magnitude higher and a sputtered rim about a factor of 5 thicker than found in typical IDPs (J. P. Bradley Pers. Comm. 1995). This particle, shown in Figure 1, is a strong candidate for a Kuiper Belt IDP. However, any single IDP might be trapped in a planetary gravitational resonance long enough to receive this irradiation, thus detailed modeling of resonance trapping durations and probabilities, as well as the identification of additional IDPs with indications of long space exposures, will be required to convincingly identify IDPs from the Kuiper Belt.

\section{References}

Bibring, J. P et al. 1972, Science, 175, 753

Bradley, J. P. \& Brownlee, D. E. 1986, Science, 231, 1542

Christopherson, R. \& McKay, D. 1995, Lunar \& Planetary Science XXVI, 245

Cochran, A. L., Levison, H. F., Stern, S. A., \& Duncan, M. J. 1995, Ap.J., in press.

Dermott, S. F. et al. 1994, in Asteroids, Comets, and Meteors 1993, A. Milani et al. eds., Dordrecht: Kluwer, 153

Flynn, G.J. 1989, Icarus, 77, 287

Flynn, G. J. 1992, in Asteroids, Comets, and Meteors 1991, A. W. Harris \& E. Bowell eds., Houston: LPI Publication Services, 195

Flynn, G. J. 1994, Lunar \& Planetary Science XXV, 379

Humes, D. H. 1974, J. Geophys. Res., 79, 3677

Humes, D. H. 1980, J. Geophys. Res., 85, 5841

Jackson, A. A. \& Zook, H. A. 1992, Icarus, 97, 70

Jewitt, D. \& Luu, J. 1993, Nature, 362, 730

Jewitt, D. \& Luu, J. 1995, Astron. J., 109, 1867

Liou, J. C. et al. 1995, Lunar \& Planetary Science XXVI, 853

Liou, J. C. et al. 1996, these proceedings

Love, S. G. \& Brownlee, D. E. 1993, Science, 262, 550

Nishiizumi, K. et al. 1991, Earth and Planet. Sci. Lett., 104, 315

Sandford, S. A. \& Bradley, J. P. 1990, Icarus, 82, 146 